\title{
Circular RNA alterations are involved in resistance to avian leukosis virus subgroup-J-induced tumor formation in chickens
}

\author{
Xinheng Zhang ${ }^{1,2,5,6}$, Yiming Yan ${ }^{1,2,5}$, Xiaoya Lei ${ }^{1,2,5}$, Aijun $\mathrm{Li}^{3}$, Huanmin Zhang ${ }^{4}$, \\ Zhenkai Dai ${ }^{1,2,5}$, Xinjian Li ${ }^{1,2,5}$, Weiguo Chen ${ }^{1,2,5,6}$, Wencheng Lin ${ }^{1,2,5,6}$, Feng Chen ${ }^{1,2,5,6}$, \\ Jingyun $\mathrm{Ma}^{1,2,5,6}$ and Qingmei $\mathrm{Xie}^{1,2,5,6}$ \\ ${ }^{1}$ College of Animal Science, South China Agricultural University, Guangzhou, 510642, P.R. China \\ ${ }^{2}$ Guangdong Provincial Key Lab of Agro-Animal Genomics and Molecular Breeding, Guangzhou, 510642, P.R. China \\ ${ }^{3}$ College of Science and Engineering, Jinan University, Guangzhou, 510632, P.R. China \\ ${ }^{4}$ USDA, Agriculture Research Service, Avian Disease and Oncology Laboratory, East Lansing, MI, 48823, USA \\ ${ }^{5}$ Key Laboratory of Animal Health Aquaculture and Environmental Control, Guangdong, Guangzhou, 510642, P.R. China \\ ${ }^{6}$ South China Collaborative Innovation Center for Poultry Disease Control and Product Safety, Guangzhou, 510642, P.R. China \\ Correspondence to: Qingmei Xie, email: qmx@scau.edu.cn \\ Keywords: circular RNA, ALV-J-resistant chickens, circular-seq, tumor \\ Received: November 25, $2016 \quad$ Accepted: March 08, $2017 \quad$ Published: March 22, 2017 \\ Copyright: Zhang et al. This is an open-access article distributed under the terms of the Creative Commons Attribution License (CC-BY), \\ which permits unrestricted use, distribution, and reproduction in any medium, provided the original author and source are credited.
}

\section{ABSTRACT}

Avian leukosis virus subgroup (ALV-J) is an oncogenic neoplasm-inducing retrovirus that causes significant economic losses in the poultry industry. Recent studies have demonstrated circular RNAs (circRNAs) are implicated in pathogenic processes; however, no research has indicated circRNAs are involved in resistance to disease. In this study, over 1800 circRNAs were detected by circRNA sequencing of liver tissues from ALV-J-resistant $(n=3)$ and ALV-J-susceptible chickens $(n=3)$. 32 differentially expressed circRNAs were selected for analyzing including 12 upregulated in ALV-J-resistant chickens and 20 upregulated in ALV-J-susceptible chickens, besides, the top five microRNAs (miRNAs) for 12 upregulated circRNAs in ALV-J-resistant chickens were analyzed. Gene ontology and KEGG pathway analyses were performed for miRNA target genes, the predicted genes were mainly involved in immune pathways. This study provides the first evidence that circRNA alterations are involved in resistance to ALV-J-induced tumor formation. We propose circRNAs may help to mediate tumor induction and development in chickens.

\section{INTRODUCTION}

Circular RNAs (circRNAs) are recently discovered noncoding RNAs that are highly represented in the eukaryotic transcriptome [1]. Previously regarded as splicing error byproducts [2], circRNAs are mainly formed by back-splicing of covalently jointed 3 '-and 5 '-ends [3]. Abundant expression of circRNAs that compete with endogenous RNAs has been reported [4]. To date, three types have been reported: circular intronic RNA, exonic circRNA and retained-intron circRNA [5-7]. Several functions have been identified for circRNAs, including RNA transport [8], protein binding [9] and regulation of translation [5]. In 2013, circRNAs were identified as efficient microRNA (miRNA) sponges [10, 11].
Endogenous linear RNAs have the ability to sequester and inhibit miRNA activity [12], indicting the existence of close interactions between circRNAs, miRNAs and mRNAs. Moreover, circRNAs have been associated with diseases, including Alzheimer disease, colorectal and ovarian cancer, idiopathic lung fibrosis and hepatocellular carcinoma [13-15].

The oncogenic retrovirus avian leukosis virus (ALV) causes a major infectious disease that leads to poor egg laying performance in chickens and impacts the poultry industry worldwide [16]. There are seven subgroups of ALV in chickens: ALV-A, -B, -C, -D, -E, -J and $-\mathrm{K}[17,18]$. ALV-J was first reported in commercial meat-type chickens in England in 1988 [19]. ALV infection spreads by horizontal transmission, vertical or 
congenital transmission and genetic transmission in flocks $[20,21]$. Chickens infected with ALV-J can develop immunosuppression together with diverse tumors, such as myelocytomas, sarcomas, hemangiomas, nephromas and erythroblastosis, as well as myeloid leukosis, and the virus induces high mortality [22-24]. Up to now, no effective vaccine has been developed for ALV-J. The control and eradication of ALV-J from pedigree generations has become a priority in the breeder industry [25]. Breeding chickens that are naturally resistant to ALV-J infection is one potential control measure.

In a previous study, we bred ALV-J-resistant and -susceptible chickens and identified an anti-tumor gene by RNA sequencing [17]. To assess whether circRNAs are involved in resistance to ALV-J-induced tumor formation, tissue samples from ALV-J-resistant and -susceptible chickens were subjected to circRNA sequencing. The study lays the foundation of circRNAs may be used as a molecular marker of ALV-J-resistance in chickens and indicates circRNAs could play a role in cancer in chickens.

\section{RESULTS}

\section{CircRNAs expression profiles}

In total, the numbers of reads from the three liver tissue samples from ALV-J-resistant chickens were 71314622,69193854 and 71259856, resulting in mapped read counts of 55805408, 55911773 and 56634896 respectively (Figure 1A). The numbers of reads from the three tissue samples from ALV-J-susceptible chickens were $65310898,64652876,65505628$, resulting in mapped read counts of 52943856, 52223375 and 52764699 respectively. Overall, over 1800 circRNAs were detected by circRNA sequencing; the Circos figure for the circRNAs in ALVJ-resistant and -susceptible chickens is shown in Figure 1B. In total, 32 significantly differentially expressed circRNAs were identified in ALV-J-resistant chickens compared to ALV-J-susceptible chickens. A heat map of the 32 differentially expressed circRNAs was generated to illustrate the distinguishable circRNA expression profiling of the samples (Figure 2); As shown in heat map, the number of $0.0,1.83$ and 2.91 on the top represents each circRNA expression level. The each row represents each circRNA and each column represents one sample. Dendrograms from clustering analysis of the samples are exhibited on the top. The left dendrogram separates the expression profiles of the resistant chickens group from the susceptible chickens group. Hierarchical clustering demonstrated the circRNA sequencing data was reliable.

\section{Differentially expressed circRNAs in ALV-J-resistant chickens}

Of the 32 significantly differentially expressed circRNAs, 12 circRNAs were upregulated and 20 were downregulated in ALV-J-resistant chickens compared to ALV-J-susceptible chickens. The 12 upregulated circRNAs in ALV-J-resistant chickens were ranked by fold change (FC), and the names of the best predicted transcript (parent gene) for each of these 12 circRNAs are shown in Table 1.

\section{The function analysis of miRNA target genes}

GO annotation of the miRNA target genes predicted to be targeted by the 12 circRNAs that were upregulated in ALV-J-resistant chickens revealed the target genes were mainly involved in immune pathways (Figure 3), such as regulation of $\mathrm{B}$ cell activation, the immune responseactivating cell surface receptor signaling pathway, B cell differentiation, alpha-beta $\mathrm{T}$ cell activation and positive regulation of mononuclear cell proliferation. KEGG pathway analysis of the predicted miRNA target genes is shown in Figure 4, three of the predicted miRNA target genes are associated with the mTOR signaling pathway.

\section{Validation of circRNA expression by qRT-PCR}

Two randomly selected circRNAs that were upregulated in ALV-J-resistant chickens (chr1:194735582194744050- and chr23:5549365-5549645-) and two randomly circRNAs that were downregulated in ALVJ-resistant chickens (chr8:1033880-1047222- and chr5:58225735-58230830-) were selected to validate the expression levels of the significantly differentially expressed circRNAs by quantitative real-time polymerase chain reaction (qRT-PCR). The qRT-PCR data for the four circRNAs was consistent with the trends observed using circRNA sequencing (Figure 5).

\section{miRNA binding sites prediction and circRNA- targeted miRNA-gene network}

CircRNAs with high number of target sites for a specific miRNA, the top 5 predicted miRNA targets of each circRNA were listed in Table 2. We selected 3 differentially up-regulated circRNAs (chr3:43666306-43724228-, chr9:22282452-22289593and chr3:105132799-105136715-) and constructed the CircRNA-targeted miRNA-gene network. Each circRNA had 5 predicted miRNA targets, every miRNA target had 10 predicted genes (Figure 6). The network showed that circRNA-targeted miRNA-gene had a strong connection with each other.

\section{DISCUSSION}

ALV-J is a major pathogen with diverse pathotypes that leads to enormous economic losses. ALV-J has rapidly evolved with respect to its pathogenicity, host range and range of tumors induced [26]. ALV-J-induced tumors among layer-type chickens are one of the most important 
Table 1: The twelve significantly upregulated circRNAs linked to resistance to ALV-J induced tumor formation in chickens

\begin{tabular}{|c|c|c|c|c|c|c|}
\hline CircRNA ID & $P$-value & Fold change & chrom & txStart & txEnd riginal & Original Gene \\
\hline $\begin{array}{l}\text { chr13:15110945- } \\
15122920-\end{array}$ & 0.0025 & 5.8790 & $\operatorname{chr} 13$ & 15110944 & 15122920 & JADE2 \\
\hline chr9:22282452-22289593- & 0.0050 & 4.2395 & $\operatorname{chr} 9$ & 22282451 & 22289593 & LEKR1 \\
\hline chr19:6585504-6585731+ & 0.0062 & 4.0622 & $\operatorname{chr} 19$ & 6585503 & 6585731 & UbI \\
\hline $\begin{array}{l}\text { chr3:105132799- } \\
105136715-\end{array}$ & 0.0297 & 3.8360 & $\operatorname{chr} 3$ & 105132798 & 105136715 & NCOA1 \\
\hline $\begin{array}{l}\text { chr1:179476699- } \\
179479760+\end{array}$ & 0.0114 & 3.4851 & $\operatorname{chr} 1$ & 179476698 & 179479760 & KDELC2 \\
\hline chr15:469774-490727+ & 0.0166 & 3.2521 & $\operatorname{chr} 15$ & 469773 & 490727 & PPM1F \\
\hline $\begin{array}{l}\text { chr1:194735582- } \\
194744050-\end{array}$ & 0.0291 & 3.1591 & $\operatorname{chr} 1$ & 194735581 & 194744050 & RAB6A \\
\hline $\begin{array}{l}\text { chr18:11114003- } \\
11114452-\end{array}$ & 0.0367 & 2.9598 & $\operatorname{chr} 18$ & 11114002 & 11114452 & CASKIN2 \\
\hline chr13:2807735-2827999- & 0.0156 & 2.8263 & $\operatorname{chr} 13$ & 2807734 & 2827999 & RANBP17 \\
\hline chr3:43666306-43724228- & 0.0265 & 2.7662 & $\operatorname{chr} 3$ & 43666305 & 43724228 & PACRG \\
\hline chr23:5549365-5549645- & 0.0334 & 2.6458 & $\operatorname{chr} 23$ & 5549364 & 5549645 & HMGCL \\
\hline chr2:816575-817682+ & 0.0031 & 2.5679 & $\operatorname{chr} 2$ & 816574 & 817682 & SMARCC1 \\
\hline
\end{tabular}

problems facing the global poultry industry [27]. Although the tumorigenic and pathogenic mechanisms of ALV-J remain unclear and are hot topics of research, no vaccine has been developed to control this disease; therefore, assessment of the host to identify genes associated with resistance to ALV-J-induced tumors may provide a novel method of controlling this problem. In this respect, we previously bred ALV-J-resistant and -susceptible chickens and discovered an anti-tumor gene by RNA sequencing [17].

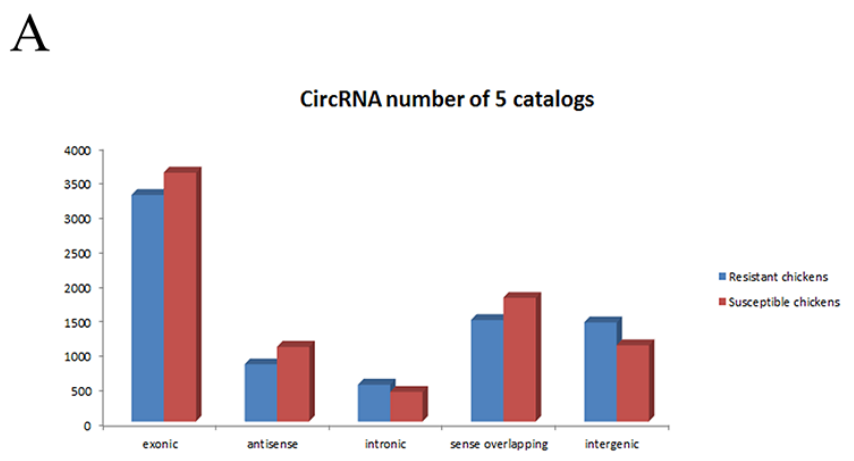

CircRNAs are a recently discovered type of noncoding RNA that have attracted significant attention [28]. Numerous studies have identified hundreds and thousands of different circRNAs, mostly comprising one to several exons of protein coding genes [14], and circRNAs have been shown to regulate gene expression in mammals [9]. A recent publication indicated that circular RNA was enriched and stable in exosomes and could be a promising biomarker for cancer diagnosis [29], besides, Circular RNA expression alterations were also involved in disease

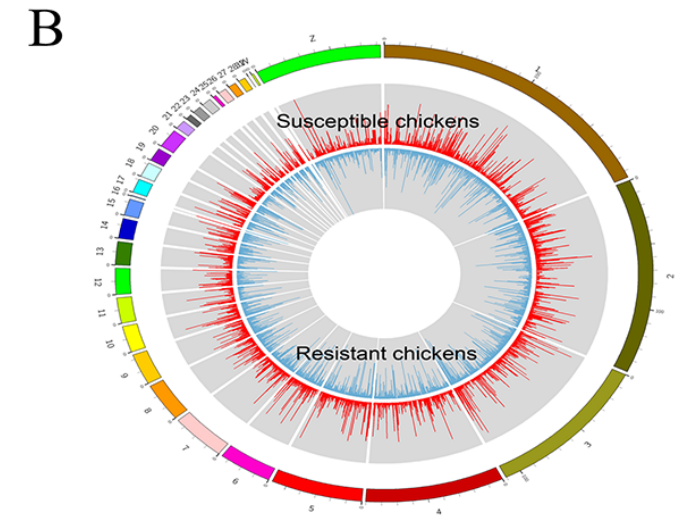

Figure 1: CircRNA sequencing data. (A) Mapping region distribution within read alignments; (B) Circos plots, outline is the reference genome, inside is the chromosome coverage across all samples. 


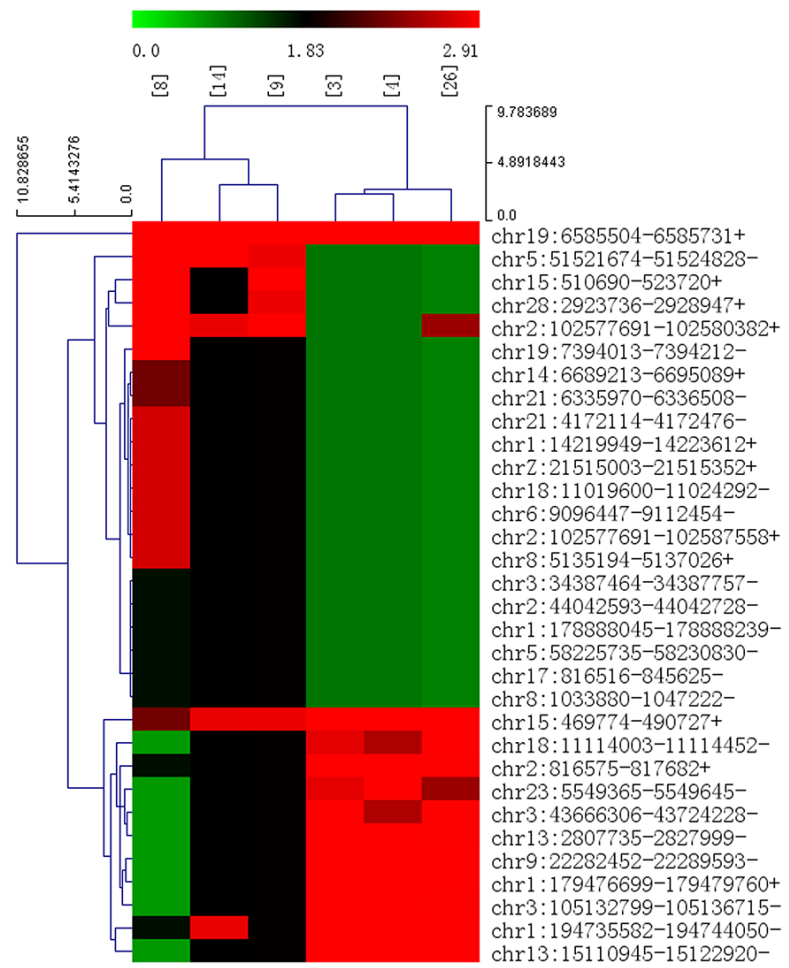

Figure 2: Heap map for the 32 significant differentially expressed circRNAs in ALV-J-resistant chickens. Sample numbers [4], [3] and [26] represent ALV-J-resistant chickens; [8], [14] and [9] represent ALV-J-susceptible chickens. The numbers 0.0, 1.83 and 2.91 on the top represent the expression levels of the circRNAs. Each row represents a circRNA and each column represents one sample. Dendrograms produced by clustering analysis of the samples are shown on the top. Twelve circRNAs were upregulated (red) and 20 were downregulated (green) in ALV-J-resistant chickens compared to ALV-J-susceptible chickens.

\section{Sig Go terms of miRNA target genes-biological process}

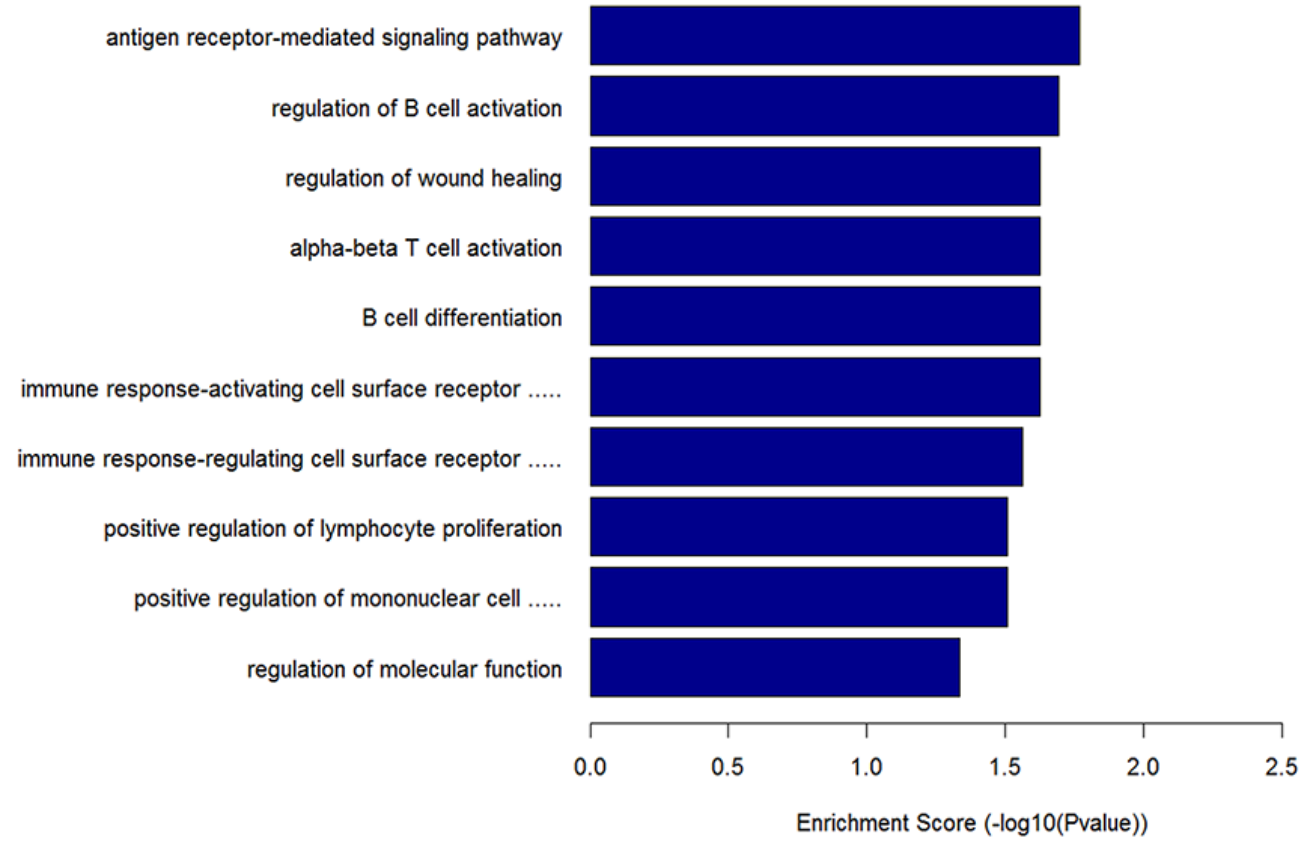

Figure 3: Annotated significant pathways targeted by the miRNA target genes of 12 circRNAs upregulated in ALV-Jresistant chickens. The horizontal axis is the $-\operatorname{LgP}$ (logarithm of $P$-value) for the pathway and the vertical axis is the pathway category. $P<0.05$ was considered significant. 


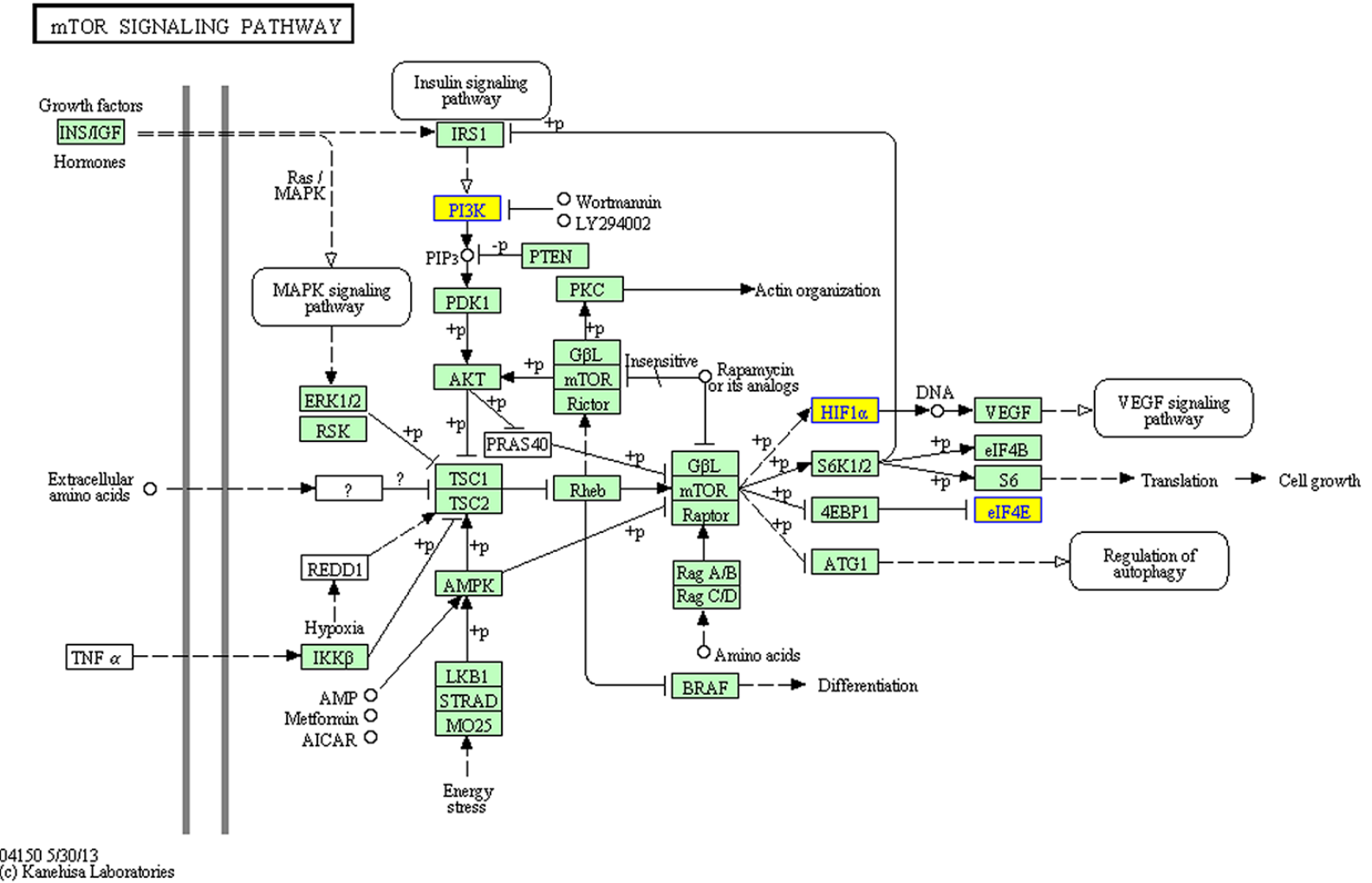

Figure 4: KEGG pathway analysis for the miRNA target genes of 12 circRNAs upregulated in ALV-J-resistant chickens. The yellow boxes indicate the genes targeted by differentially expressed circRNAs.

A

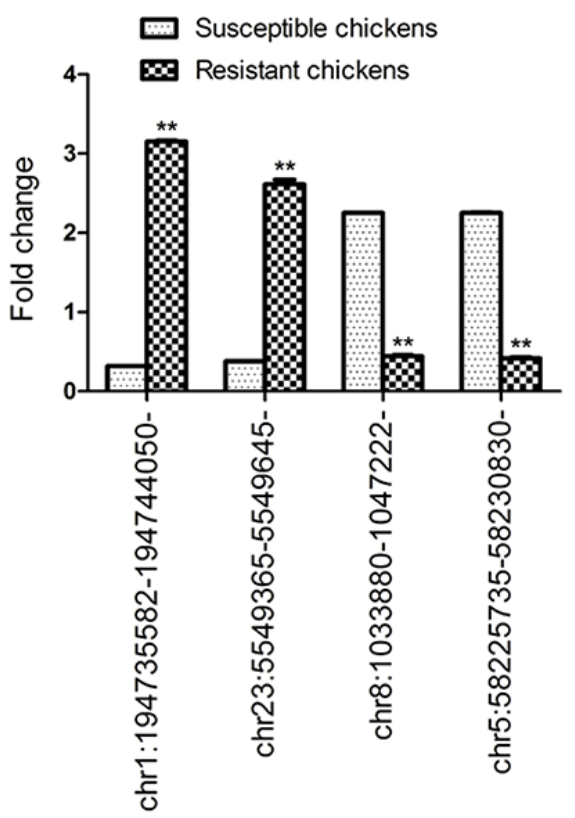

B

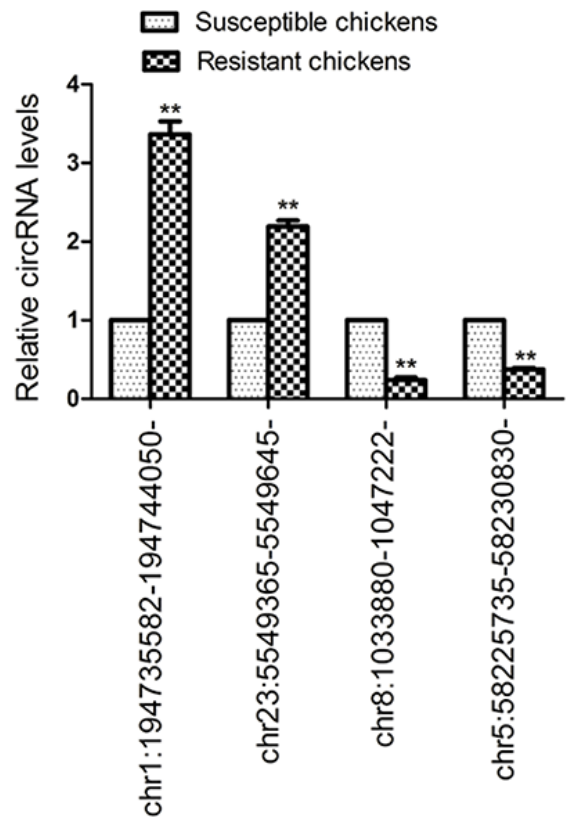

Figure 5: Validation of the expression of four selected differentially expressed circRNAs identified by circRNA sequencing. (A) Fold change for each circular RNA as indicated by circRNA sequencing; (B) Relative circRNA levels as indicated by qPCR. Bars are mean $\pm \mathrm{SD}(\mathrm{n}=3) ; * * P<0.05$. 
occur and the detailed analysis methods of circRNA alterations were also illustrated [30]. MiRNAs are highly conversed during evolution and negatively regulate target mRNAs. Moreover, a previous study demonstrated that miRNAs play critical roles in tumor formation in chickens infected with ALV-J [31]. However, there is no data on the role of circRNAs in resistance to tumor formation induced by ALV-J or other pathogens in any species. In the current study, we assessed whether circRNAs are involved in resistance to ALV-J-induced tumor formation by analyzing circRNA expression in ALV-J-resistant and -susceptible chickens [17] using circRNA sequencing.

Gene ontology (GO) is a widely used bioinformatics concept that unifies the genes and gene products of all species [32]. High-throughput circRNA sequencing and real-time PCR are standardized approaches used to assess circRNA expression; these methods have been widely used to infer host gene expression in response to disease [33, 34]. Our study demonstrates circRNAs are differentially expressed in ALV-J-resistant chickens, including the 12 upregulated circRNAs, indicating circRNAs are involved in resistance to ALV-J-induced tumor formation. In 2011, an endogenous circRNA was found to be highly expressed in human and mouse brain tissues [35], but the function of this circRNA remained unknown. In 2013, circRNAs were identified as efficient microRNA (miRNA) sponges [10]. Overexpressing miRNA target site concatamers (miRNA sponges) results in loss of miRNA function accompanied by increased endogenous target gene expression [11]. Considering that circRNA can absorb miRNA and Go terms of miRNA target genes of 12 differentially expressed circRNAs that were upregulated in ALV-J-resistant chickens in this study are linked to immune pathways such as antigen receptor signaling pathway and regulation of B cell activation, besides, three of the predicted miRNA target genes in KEGG pathway are associated with the mTOR signaling pathway which is a key regulator of cell growth and

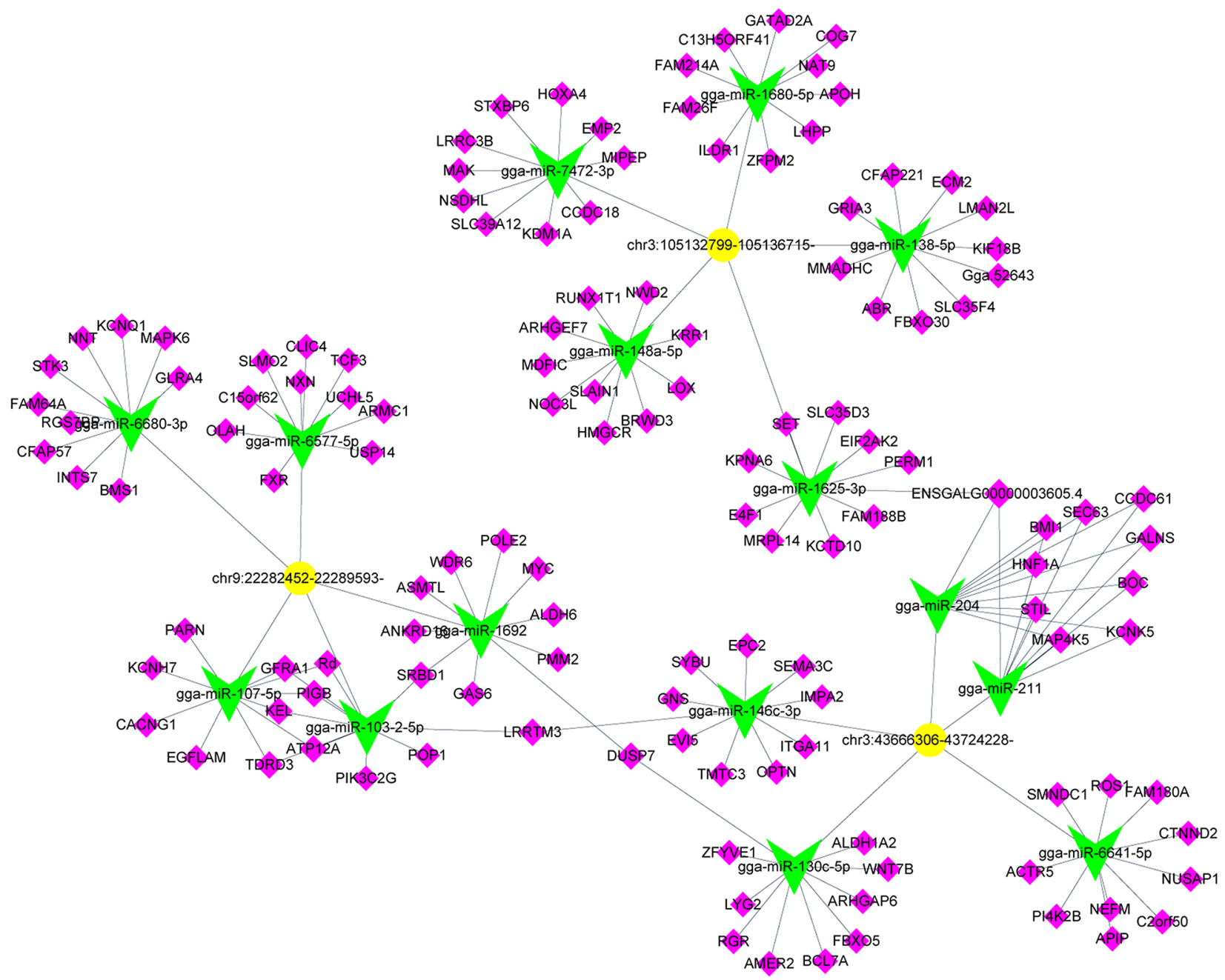

Figure 6: Predicted biomathematical circRNA-miRNA-gene network for three selected upregulated circRNAs in ALVJ-resistant chickens. Yellow represents circRNAs, green represents miRNAs and pink represents miRNA target genes. 
Table 2: Predicted miRNAs for 12 differentially expressed circRNAs linked to resistance to ALV-J induced tumor formation in chickens

\begin{tabular}{|c|c|c|c|c|c|}
\hline CircRNA ID & miRNA & miRNA & miRNA & miRNA & miRNA \\
\hline $\begin{array}{l}\text { chr13:15110945- } \\
\text { 15122920- }\end{array}$ & gga-miR-6634-5p & gga-miR-1649-5p & gga-miR-24-3p & gga-miR-6587-3p & gga-miR-1663-3p \\
\hline $\begin{array}{l}\text { chr9:22282452- } \\
\text { 22289593- }\end{array}$ & gga-miR-103-2-5p & gga-miR-107-5p & gga-miR-6680-3p & gga-miR-1692 & gga-miR-6577-5p \\
\hline $\begin{array}{l}\text { chr19:6585504- } \\
6585731+\end{array}$ & gga-miR-3530-3p & gga-miR-6549-5p & gga-miR-1664-5p & gga-miR-1764-5p & gga-miR-193a-5p \\
\hline $\begin{array}{l}\text { chr3:105132799- } \\
105136715-\end{array}$ & gga-miR-7472-3p & gga-miR-138-5p & gga-miR-1625-3p & gga-miR-148a-5p & gga-miR-1680-5p \\
\hline $\begin{array}{l}\text { chr1:179476699- } \\
179479760+\end{array}$ & gga-miR-6632-5p & gga-miR-153-5p & gga-miR-1705 & gga-miR-1684b-3p & gga-miR-6698-3p \\
\hline $\begin{array}{l}\text { chr15:469774- } \\
490727+\end{array}$ & gga-miR-2129 & gga-miR-1632-5p & gga-miR-1659 & gga-miR-6698-3p & gga-miR-6598-5p \\
\hline $\begin{array}{l}\text { chr1:194735582- } \\
194744050-\end{array}$ & gga-miR-6660-3p & gga-miR-1662 & gga-miR-15c-5p & gga-miR-15b-5p & gga-miR-1787 \\
\hline $\begin{array}{l}\text { chr18:11114003- } \\
\text { 11114452- }\end{array}$ & gga-miR-6607-5p & gga-miR-1749-3p & gga-miR-1785 & gga-miR-19b-5p & gga-miR-6545-3p \\
\hline $\begin{array}{l}\text { chr13:2807735- } \\
\text { 2827999- }\end{array}$ & gga-miR-365b-5p & gga-miR-1756b & gga-miR-217-3p & gga-miR-6690-5p & gga-miR-1771 \\
\hline $\begin{array}{l}\text { chr3:43666306- } \\
43724228-\end{array}$ & gga-miR-146c-3p & gga-miR-130c-5p & gga-miR-211 & gga-miR-204 & gga-miR-6641-5p \\
\hline $\begin{array}{l}\text { chr23:5549365- } \\
5549645-\end{array}$ & gga-miR-6702-5p & gga-miR-1662 & gga-miR-34b-5p & gga-miR-6611-5p & gga-miR-6641-5p \\
\hline $\begin{array}{l}\operatorname{chr} 2: 816575- \\
817682+\end{array}$ & gga-miR-6574-5p & gga-miR-6577-5p & gga-miR-1596-5p & gga-miR-1786 & gga-miR-1615 \\
\hline
\end{tabular}

proliferation and mTOR dysregulation has a key role to play in various cancers [36], so we suspect circRNAs could be involved in initiation of the immune effects that protect ALV-J-resistant chickens from infection and tumor formation. We propose circRNAs may represent a new mediator of tumor initiation and progression.

Further study of the functions of these circRNAs could improve our understanding of the mechanisms by which circRNAs confer resistance against ALV-J-induced tumor formation. This data also provides a basis for assessing the potential of the differentially expressed circRNAs as novel of biomarkers of resistance to ALV-J-induced tumor formation in chickens. Moreover, this study provides a solid theoretical foundation for further research into the molecular mechanisms by which circular RNAs play a role in the initiation and progression of cancer in chicken.

\section{MATERIALS AND METHODS}

\section{Ethics statement}

All experiments were carried out in strict accordance with the recommendations of the Guide for the Care and Use of Laboratory Animals of the National Institutes of Health. The use of animals in this study was approved by South China Agricultural University Committee of Animal Experiments (approval ID: 201004152).

\section{Samples and RNA isolation}

ALV-J-resistant and -susceptible chickens (F3 generation) were used as classical models of resistance or easily-induced tumors in response to ALV-J challenge [17]. The standard ALV-J NX0101 strain which induced myeloid tumors was used in this study. RNA was isolated from livers of three ALV-J-resistant and three ALV-Jsusceptible chickens at 20 weeks after challenge with ALV-J. Total RNA was isolated from each group using TRizol reagent (Life Technologies, Carlsbad, CA, USA) according to the manufacturer's instructions. The concentration of the RNA samples was determined by assessing the OD260/280 using a NanoDrop ND-2000 instrument (Thermo, Waltham, MA, USA). The integrity of the RNA samples was assessed by denaturing agarose gel electrophoresis. 


\section{RNA library construction and circRNA sequencing}

Total RNA from each sample was used to prepare the circRNA sequencing library, via the following steps: 1) $5 \mu \mathrm{g}$ total RNA samples were pretreated to enrich circRNAs using the CircRNA Enrichment Kit (Cloud-seq Inc., Shanghai, China). RNA libraries were constructed from the treated RNAs using the TruSeq Stranded Total RNA Library Prep Kit (Illumina, San Diego, CA, USA) according to the manufacturer's instructions. Libraries were controlled for quality and quantified using the BioAnalyzer 2100 system (Agilent Technologies, Inc., Santa Clara, CA, USA). The libraries were denatured as single-stranded DNA molecules, captured on Illumina flow cells, amplified in situ as clusters and finally sequenced for 150 cycles on an Illumina HiSeq Sequencer according to the manufacturer's instructions.

\section{CircRNA sequencing analysis}

Paired-end reads were harvested from the Illumina HiSeq 4000 sequencer and quality controlled using Q30 [37]. After 3' adaptor-trimming and removal of low quality reads using Cutadapt software [38], the high quality trimmed reads were used for analysis of circRNAs. The high quality reads were aligned to the reference genome/ transcriptome using bowtie 2 software and circRNAs were detected and identified using find_circ software $[39,1]$. Raw junction reads for all samples were normalized to the number of total mapped reads and $\log 2$ transformed. Circos software was used to construct the circos figure [40]. CircRNAs exhibiting fold changes $\geq 2.0$ with $P$-values $\leq 0.05$ were classified as significantly differentially expressed circRNAs.

\section{Bioinformatic analysis and target prediction}

CircRNA and miRNA interactions were predicted using customized Arraystar miRNA target prediction software based on TargetScan and miRanda [41, 42]; the top five putative target miRNAs were identified for upregulated circRNAs in ALV-J-resistant chickens. The putative target genes of these miRNAs were identified using Targetscan [41]. Cytoscape software was used to construct the circRNA-miRNA-gene networks [43]. Gene ontology (GO) and Kyoto Encyclopedia of Genes and Genomes (KEGG) analysis were performed for the differentially expressed circRNA-associated genes $[44,45]$.

\section{Quantitative real-time polymerase chain reaction analysis}

Total RNA was reverse transcribed to synthesize cDNA using PrimeScript RT Reagent Kit (Perfect Real Time; TaKaRa, Osaka, Japan) and subjected to quantitative real-time polymerase chain reaction (qRTPCR) analysis on an Applied Biosystems 7500 Fast Real-Time PCR System (Roche, Basel, Switzerland; software version 2.0.5) with SYBR Green qPCR SMix (ROX; Roche). The primers used for the qRT-PCR analysis were: chr1:194735582-194744050- (F 5'-GGG CTTGTTTCTGGTTTGGGTTAG-3', R 5'-AGTGTCCAT TGAGGAAGGAGAAAG-3'), chr23:5549365-5549645(F 5'-CTCCCTGTCTCCTCTCCCAATCTT-3', R 5'-CTC TGGTTCCTCTGTACTGCTGTATCC-3'), chr8:10338801047222- (F 5'-GTTCCCCTAAGAAGCATTTGACAGA CT-3', R 5'-GGGATTAGAAGGAACTCCAGACACG -3'), chr5:58225735-58230830- (F 5'-CTTCAGGGCTT CATCTGCTCTC-3', R 5'-GACTCCATTACTCCAGAC AAAGTACG-3'), and $\beta$-actin mRNA (F 5'-GAAGT ACCCCATTGAACACGG-3', R 5'-AGGCATACAGGGA CAGCACA-3'). Three independent samples were analyzed for each group; all samples were assessed in triplicate. The $2^{-\triangle \Delta \mathrm{Ct}}$ method was used to analyze the $\mathrm{qPCR}$ results [46].

\section{Statistical analysis}

Data are expressed as mean \pm standard deviation (SD). The Student's $t$-test was used to assess differences among groups; $P<0.05$ was considered significant.

\section{Author contributions}

Xinheng Zhang conducted the experiments and wrote the manuscript; Qingmei Xie modified the manuscript, and supervised the whole work; Yiming Yan, Xiaoya Lei, ZhenKai Dai and Xinjian Li conducted part of the experiments; Weiguo Chen and Wencheng Lin analyzed part of the data; Feng Chen and Jingyun Ma provided important suggestions; Huanmin Zhang and Aijun Li provided guidance on experimental design and data analysis.

\section{ACKNOWLEDGMENTS}

The authors would like to thank Professor Huanmin Zhang (USDA, Agriculture Research Service, Avian Disease and Oncology Laboratory) for providing guidance on experimental design and thank Professor Aijun Li for providing guidance on data analysis.

\section{CONFLICTS OF INTEREST}

The authors declare no conflicts of interest.

\section{GRANT SUPPORT}

This study was supported by the National Natural Science Foundation of China (Grant Nos. 31672564, 31472217), Construction project of modern agricultural 
science and technology innovation alliance in Guangdong Province (2016LM1112), International Science and technology cooperation project of Guangdong Province (2016A050502042) and the Natural Science Foundation of Guangdong Province (Grant No. S2013030013313).

\section{REFERENCES}

1. Memczak S, Jens M, Elefsinioti A, Torti F, Krueger J, Rybak A, Maier L, Mackowiak SD, Gregersen LH, Munschauer M, Loewer A, Ziebold U, Landthaler M, et al. Circular RNAs are a large class of animal RNAs with regulatory potency. Nature. 2013; 495: 333-338.

2. Cocquerelle C, Mascrez B, Hetuin D, Bailleul B. Missplicing yields circular RNA molecules. FASEB J. 1993; 7: $155-160$.

3. Hentze MW, Preiss T. Circular RNAs: splicing's enigma variations. EMBO J. 2013; 32: 923-925.

4. Wang X, Zhang Y, Huang L, Zhang J, Pan F, Li B, Yan Y, Jia B, Liu H, Li S, Zheng W. Decreased expression of hsa_circ_001988 in colorectal cancer and its clinical significances. Int J Clin Exp Pathol. 2015; 8: 16020-16025.

5. Zhang Y, Zhang XO, Chen T, Xiang JF, Yin QF, Xing YH, Zhu S, Yang L, Chen LL. Circular intronic long noncoding RNAs. Mol Cell. 2013; 51: 792-806.

6. Danan M, Schwartz S, Edelheit S, Sorek R. Transcriptomewide discovery of circular RNAs in Archaea. Nucleic Acids Res. 2012; 40: 3131-3142.

7. Salzman J, Gawad C, Wang PL, Lacayo N, Brown PO. Circular RNAs are the predominant transcript isoform from hundreds of human genes in diverse cell types. PLoS One. 2012; 7: e30733.

8. Ashwal-Fluss R, Meyer M, Pamudurti NR, Ivanov A, Bartok O, Hanan M, Evantal N, Memczak S, Rajewsky N, Kadener S. circRNA biogenesis competes with pre-mRNA splicing. Mol Cell. 2014; 56; 55-66.

9. Jeck WR, Sorrentino JA, Wang K, Slevin MK, Burd CE, Liu J, Marzluff WF, Sharpless NE. Circular RNAs are abundant, conserved, and associated with ALU repeats. RNA. 2013; 19: 141-157.

10. Hansen TB, Jensen T, Clausen BH, Bramsen JB, Finsen B, Damgaard CK, Kjems J. Natural RNA circles function as efficient microRNA sponges. Nature. 2013; 495: 384-388.

11. Ebert MS, Neilson JR, Sharp PA. MicroRNA sponges: competitive inhibitors of small RNAs in mammalian cells. Nature Methods. 2007; 4: 721-726.

12. Chi SW, Zang JB, Mele A, Darnell RB. Argonaute HITSCLIP decodes microRNA-mRNA interaction maps. Nature. 2009; 460: 479-486.

13. Lukiw WJ. Circular RNA (circRNA) in Alzheimer's disease (AD). Front Genet. 2013; 4: 307.

14. Bachmayr-Heyda A, Reiner AT, Auer K, Sukhbaatar N, Aust S, Bachleitner-Hofmann T, Mesteri I, Grunt TW, Zeillinger
R, Pils D. Correlation of circular RNA abundance with proliferation--exemplified with colorectal and ovarian cancer, idiopathic lung fibrosis, and normal human tissues. Sci Rep. 2015; 5: 8057.

15. Shang X, Li G, Liu H, Li T, Liu J, Zhao Q, Wang C. Comprehensive Circular RNA Profiling Reveals That hsa circ_0005075, a New Circular RNA Biomarker, Is Involved in Hepatocellular Carcinoma Development. Medicine (Baltimore). 2016; 95: e3811.

16. Liu XL, Shan WJ, Jia LJ, Yang X, Zhang JJ, Wu YR, Xu FZ, Li JN. Avian leukosis virus subgroup J triggers caspase1 -mediated inflammatory response in chick livers. Virus Res. 2016; 215: 65-71.

17. Zhang X, Yan Z, Li X, Lin W, Dai Z, Yan Y, Lu P, Chen W, Zhang H, Chen F, Ma J, Xie Q. GADD45 $\beta$, an anti-tumor gene, inhibits avian leukosis virus subgroup J replication in chickens. Oncotarget. 2016; 7:68883-68893. doi: 10.18632/ oncotarget. 12027.

18. Li X, Lin W, Chang S, Zhao P, Zhang X, Liu Y, Chen W, Li B, Shu D, Zhang H, Chen F, Xie Q. Isolation, identification and evolution analysis of a novel subgroup of avian leukosis virus isolated from a local Chinese yellow broiler in South China. Arch Virol. 2016; 161: 2717-2725.

19. Payne LN, Brown SR, Bumstead N, Howes K, Frazier JA, Thouless ME. A novel subgroup of exogenous avian leukosis virus in chickens. J Gen Virol. 1991; 72: 801-807.

20. Payne LN, Nair V. The long review: 40 years of avian leukosis research. Avian Pathol. 2012; 41: 11-19.

21. Li Y, Liu X, Liu H, Xu C, Liao Y, Wu X, Cao W, Liao M. Isolation, identification, and phylogenetic analysis of two avian leukosis virus subgroup J strains associated with hemangioma and myeloid leukosis. Vet Microbiol. 2013; 166, 356-364.

22. Fadly A, Payne L. Leukosis/sarcoma group. In: Diseases of poultry, 11th ed. Saif YM, Barnes HJ, Glisson JR, Fadly AM, McDougald LR, Swayne DE, eds. Iowa State Press, Ames, IA. 2003; 465-516.

23. Stedman NL, Brwon TP. Body weight suppression in broilers naturally infected with avian leukosis virus subgroup J. Avian Dis. 1999; 43: 604-610.

24. Du Y, Cui Z, Qin A. Subgroup J of avian leukosis viruses in China. China Poultry. 1999; 3: 1-4.

25. McKay JC, Rosales AG. Control and eradication of ALVJ. In: International Symposium on ALV-J and Other Avian Retroviruses. World Veterinary Poultry Association, Rauischholzhausen, Germany. 2000; 248-255.

26. Wang W, Zhang ZP, Tian J, Xiao ZG, Meng QW. Inhibition of avian leukosis virus subgroup $\mathrm{J}$ replication by miRNA targeted against env. Virus Genes. 2013; 47: 34-41.

27. Xu B, Dong W, Yu C, He Z, Lv Y, Sun Y, Feng X, Li N, Lee LF, Li M. Occurrence of avian leukosis virus subgroup J in commercial layer flocks in China. Avian Pathol. 2004; 33: 13-17.

28. Sand M, Bechara FG, Gambichler T, Sand D, Bromba M, Hahn SA, Stockfleth E, Hessam S. Circular RNA expression 
in cutaneous squamous cell carcinoma. J Dermatol Sci. 2016; 83: 210-218.

29. Li Y, Zheng Q, Bao C, Li S, Guo W, Zhao J, Chen D, Gu J, He X, Huang S. Circular RNA is enriched and stable in exosomes: a promising biomarker for cancer diagnosis. Cell Res. 2015; 25: 981-984.

30. Lin SP, Ye S, Long Y, Fan Y, Mao HF, Chen MT, Ma QJ. Circular RNA expression alterations are involved in OGD/ R-induced neuron injury. Biochem Biophys Res Commun. 2016; 471: 52-56.

31. Dai Z, Ji J, Yan Y, Lin W, Li H, Chen F, Liu Y, Chen W, Bi Y, Xie Q. Role of gga-miR-221 and gga-miR-222 during tumor formation in chickens infected by subgroup $\mathrm{J}$ avian leukosis virus. Viruses. 2015; 7: 6538-6351.

32. Huntley RP, Sawford T, Mutowo-Meullenet P, Shypitsyna A, Bonilla C, Martin MJ, O'Donovan C. The GOA database: gene ontology annotation updates for 2015 . Nucleic Acids Res. 2015; 43: 1057-1063.

33. Wang H, Yang J, Yang J, Fan Z, Yang C. Circular RNAs: Novel rising stars in cardiovascular disease research. Int J Cardiol. 2016; 202: 726-727.

34. Mackay IM, Arden KE, Nitsche A. Real-time PCR in virology. Nucleic Acids Res. 2002; 30, 1292-1305.

35. Hansen TB, Wiklund ED, Bramsen JB, Villadsen SB, Statham AL, Clark SJ, Kjems J. miRNA-dependent gene silencing involving Ago2-mediated cleavage of a circular antisense RNA. EMBO J. 2011; 30: 4414-4422.

36. Advani SH. Targeting mTOR pathway: Anew concept in cancer therapy. Indian J Med Paediatr Oncol. 2010; 31: 132-136.

37. Ewing B, Hillier L, Wendl MC, Green P. Base-calling of automated sequencer traces using phred. I. Accuracy assessment. Genome Res. 1998; 8: 175-185.
38. Martin M. Cutadapt removes adapter sequences from high-throughput sequencing reads. EMBnet J. 2001; 17: 10-12.

39. Langmead B, Salzberg SL. Fast gapped-read alignment with Bowtie 2. Nat Methods. 1923; 9: 357-359.

40. Krzywinski M, Schein J, Birol I, Connors J, Gascoyne R, Horsman D, Jones SJ, Marra MA. Circos: an information aesthetic for comparative genomics. Genome Res. 2009; 19: 1639-1645.

41. Enright AJ, John B, Gaul U, Tuschl T, Sander C, Marks DS. MicroRNA targets in Drosophila. Genome Biol. 2003; 5: R1.

42. Pasquinelli AE. MicroRNAs and their targets: recognition, regulation and an emerging reciprocal relationship. Nat Rev Genet. 2012; 13: 271-282.

43. Smoot ME, Ono K, Ruscheinski J, Wang PL, Ideker T. Cytoscape 2.8: new features for data integration and network visualization. Bioinformatics. 2011; 27: 431-432.

44. Xing Z, Chu C, Chen L, Kong X. The use of Gene Ontology terms and KEGG pathways for analysis and prediction of oncogenes. Biochim Biophys Acta. 2016; 1860: 2725-2734.

45. Bkanehisa M, Sato Y, Morishima K. BlastKOALA and GhostKOALA: KEGG Tools for Functional Characterization of Genome and Metagenome Sequences. J Mol Biol. 2016; 428: 726-731.

46. Gao Y, Yun B, Qin L, Pan W, Qu Y, Liu Z, Wang Y, Qi X, Gao H, Wang $\mathrm{X}$. Molecular epidemiology of avian leukosis virus subgroup $\mathrm{J}$ in layer flocks in China. J Clin Microbiol. 2012; 50: 953-960. 